\title{
Development and Characterization of a Solid Phase for Single-Step Enrichment of Pathogenic Targets
}

\author{
M.J. Archer*, D.A. Stenger and B. Lin \\ Center for Bio/Molecular Science \& Engineering, Code 6900, Naval Research Laboratory, Washington, DC 20375, USA
}

\begin{abstract}
The identification of low abundance target nucleic acids in a complex matrix can be challenging due to the abundance background material. Current methods use two-step processes which are time consuming, prone to contamination and usually limited to one pathogen. In this study we describe a single-step target-capture approach using magnetic microbeads with capture probes covalently attached through a phosphorus dendrimer linker. This approach was also used successfully for simultaneous capturing of two low abundance pathogenic nucleic acids present in a complex matrix (800fold excess of background nucleic acids) by using a multi-pathogen solid phase. The thermal stability of the solid phase allows denaturation and capture to proceed sequentially and the recovery of the targets to be performed by heat denaturation without the risk of probe shedding. The critical variables involved in the development of the solid phase and the steps required for further optimization are discussed.
\end{abstract}

\section{INTRODUCTION}

Sequence capture of nucleic acids has been used for more than a decade in fields such as clinical diagnosis [1-4], library construction for genetic sequencing [5] and veterinary medicine [6-8] to improve assay sensitivity and specificity. In the presence of background genomic nucleic acids (sometimes one million-fold more abundant) looking for a target of interest is like looking for a needle in a hay stack, thus the main objective of sequence-capture protocols is to enrich nucleic acids of interest from background or interfering material through selective hybridization. Even when using highly specific PCR primers, excess background material can interfere with amplification (false negative) or lead to non-specific priming (false positive), thus, enriching target nucleic acids will enhance the detection specificity and sensitivity. This is particularly important for non-cultivable pathogens since molecular approaches are the main methods to identify these organisms [1].

Several sequence-capture approaches (sometimes referred to as enrichment) have been developed for this purpose. These approaches utilize similar strategies and are performed in a two-step process. First, biotinylated capture probes selective to a target or targets of interest are hybridized with samples containing background and target nucleic acids. Then, the solid phase, such as magnetic microbeads functionalized with streptavidin (SA) or neutravidin are introduced to bind the probe-target complex, while the unbound material is washed away. The magnetic microbeads with probe-target complex can then be used directly in Reverse Transcription (RT)-PCR/PCR reaction mix [1-4, 7-10] or the captured targets can be released through alkali denaturation and then be subjected to amplification [5].

*Address correspondence to this author at the Center for Bio/Molecular Science \& Engineering, Code 6900, Naval Research Laboratory, Washington DC 20375, USA; Tel: (202) 404-2522; Fax: (202) 767-9594;

E-mail: marie.archer@nrl.navy.mil
Although promising, these processes require multiple manual manipulations which are time consuming and labor intense. In many circumstances, probe-target hybridization has to be performed overnight followed by several hours of solid phase hybridization that increase the assay time significantly [4-6]. Also, the binding capacity of the magnetic beads (manufacturer's restriction) is limited which leads to incomplete binding with large amounts of capture probe [3]. Furthermore, it has been shown that amplifications can be inhibited, reduced or even prevented by small alterations to process parameters such as lengthening the bead residence time in the magnetic separator, excessive mixing, and changes in the salt concentration or $\mathrm{pH}$ [6]. In order to avoid inhibition of the amplification step, only half of the total amount of the beads used for each capture can be used per amplification reaction [3]. This effectively reduces the availability of the capture targets and is counterproductive to the purpose of enrichment. A single-step protocol that could eliminate these restrictions would be desirable. The attempts made so far for a direct capture of the targets using the biotin-SA interaction have not performed well as the capture efficiency was significantly lower than a twostep approach $[3,5,9]$. While low capture efficiency of these single-step protocols can be attributed to several factors such as steric hindrance, limited diffusion of targets, or the presence of endogenous biotin in the samples $[3,11,12]$, the dominant factor causing low capture efficiency is the lack of stability of the biotin-avidin links at high temperature in the presence of surfactants such as SDS (essentials constituents of the hybridization buffer) $[11,12]$.

In this paper we present the development of a solid phase for single-step sequence-capture applications that utilizes a capture probe covalently linked to magnetic beads that overcomes the limitations of the biotin-avidin linkage. Our previous study focused on background subtraction demonstrated that phosphorous dendrimers as linkers provide the thermal stability required in a single-step capture without the risk of probe shedding. Phosphorus dendrimers are bowl-shaped inorganic molecules that bear aldehyde terminated branches in 
their periphery. Based on its size and the number of branches available for covalent attachment, various generations of phosphorus dendrimers are available to couple onto the magnetic microbeads through reaction of the aldehyde groups with the primary amines present on the surface of the magnetic microbeads (2-3 um diameter). The functionalized magnetic beads then provide aldehyde branches for immobilizing amino-labeled DNA capture probes. Our results also demonstrated that the linker characteristics (size, and number of branches available for covalent binding) and the length of the probes affect the capturing efficiency [13]. Based on this knowledge, we sought to demonstrate the potential of this approach for sequence-capture of pathogenic nucleic acids using influenza A/H1N1 (FluA H1N1) virus and human adenovirus serotype 4 (HAdV-4) as model organisms. Furthermore, it is desirable to enrich for multiple pathogens simultaneously and therefore the presence of more than one capture probe with different lengths and base composition (a "multipathogen" solid phase for enrichment) is required. Here, we also demonstrate the feasibility of this concept through the simultaneous enrichment of FluA H1N1 and HAdV-4 from a matrix containing an 800-fold excess background material and address the critical variables, such as the capture probe specificity, solid phase characteristics and assay conditions that affect the performance and have to be considered for further optimization.

\section{MATERIALS AND METHODS}

All the primers used in this study were synthesized by Operon Biotechnology (Huntsville, AL) and listed in supplemental Tables $\mathbf{1}$ and $\mathbf{2}$. All the amplification steps were carried out in Peltier Thermal Cycler- PTC 240 DNA Engine Tetrad 2 (MJ Research Inc, Reno, NV).

Random capture probes synthesis. For the HAdV-4, DNA template was digested with EcoRI (New England Biolabs Inc. Ipswich, MA) in a $50 \mu 1$ reaction volume and concentrated to $25 \mu 1$ using ChargeSwitch ${ }^{\circledR}$ PCR Clean-Up Kit (Invitrogen Life Technologies, Carlsbad, CA). The fragmented template was randomly amplified using primers L and LN (Supplemental Table 1). The PCR reaction was carried out in a $50 \mu \mathrm{l}$ volume containing $20 \mathrm{mM}$ Tris- $\mathrm{HCl}$ ( $\mathrm{pH} 8.4), 50 \mathrm{mM} \mathrm{KCl}, 3 \mathrm{mM}$ $\mathrm{MgCl}_{2}, 200 \mu \mathrm{M}$ dNTPs, $1 \mu \mathrm{M}$ primer L, $800 \mathrm{nM}$ primer LN, 2 $\mathrm{U}$ of Platinum Taq DNA Polymerase (Invitrogen) and $50 \mathrm{ng}$ of template. The amplification reaction was carried out with preliminary denaturation at $95^{\circ} \mathrm{C}$ for $3 \mathrm{~min}$. followed by 5 cycles of: $94^{\circ} \mathrm{C}$ for $30 \mathrm{sec} ., 25^{\circ} \mathrm{C}$ for $90 \mathrm{sec} ., 72^{\circ} \mathrm{C}$ for $60 \mathrm{sec}$, and 35 cycles of $94^{\circ} \mathrm{C}$ for $30 \mathrm{sec}$. and $64^{\circ} \mathrm{C}$ for $60 \mathrm{sec}$. The products were purified using Qiagen PCR purification kit (Qiagen, Inc. Valencia, CA) and eluted in nuclease free water.

For the FluA H1N1, DNA template was obtained using a single step $25 \mu$ RT-PCR reaction containing $0.2 \mathrm{mM}$ of each dNTP, $3 \mathrm{mM} \mathrm{MgSO}$, $200 \mathrm{mM}$ each of AMPforl and AMP rev1 primers [14], $20 \mathrm{U}$ RNaseOUT (Invitrogen), $0.5 \mathrm{ul}$ of ThermoScript Plus/Platinum Taq Mix (Invitrogen) and $5 \mathrm{ng}$ of FluA H1N1 RNA. The RT-PCR reaction was carried out with initial incubation at $50^{\circ} \mathrm{C}$ for $30 \mathrm{~min}$. followed by $95^{\circ} \mathrm{C}$ for 5 min., and 35 cycles of $95^{\circ} \mathrm{C}$ for $60 \mathrm{sec}$., $50^{\circ} \mathrm{C}$ for $60 \mathrm{sec}$., and $72^{\circ} \mathrm{C}$ for $2 \mathrm{~min}$., final extension was done at $72^{\circ} \mathrm{C}$ for $10 \mathrm{~min}$. The products were purified using Qiagen PCR purification kit (Qiagen) and eluted in nuclease free water then used for ran-
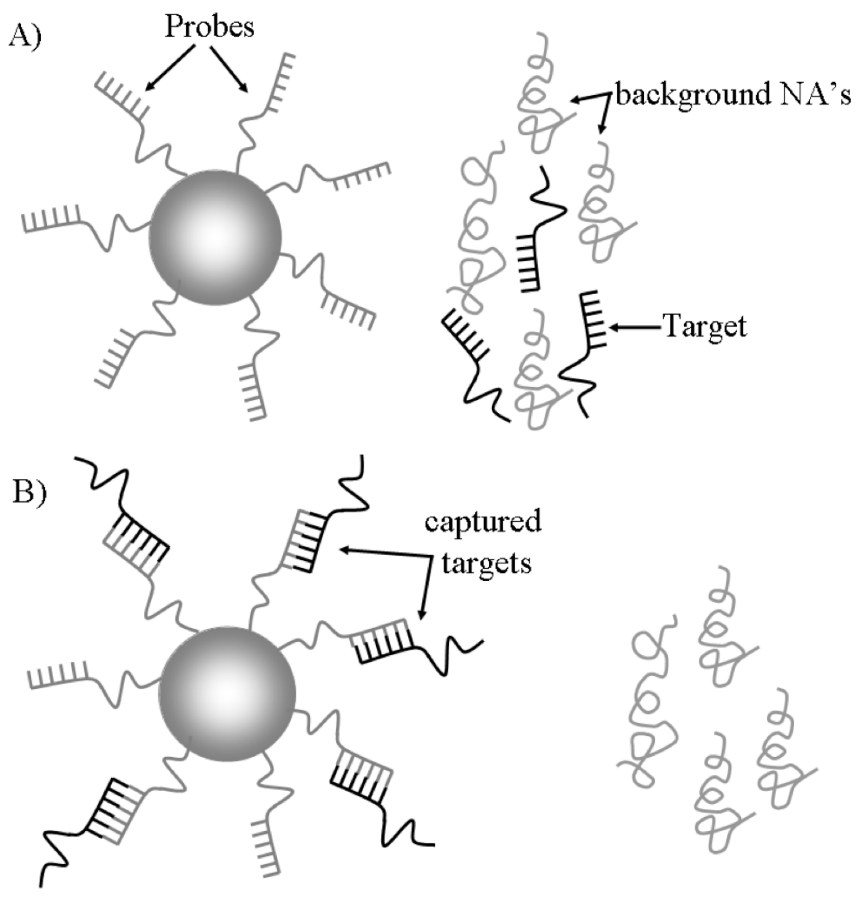

Unbound background is discarded

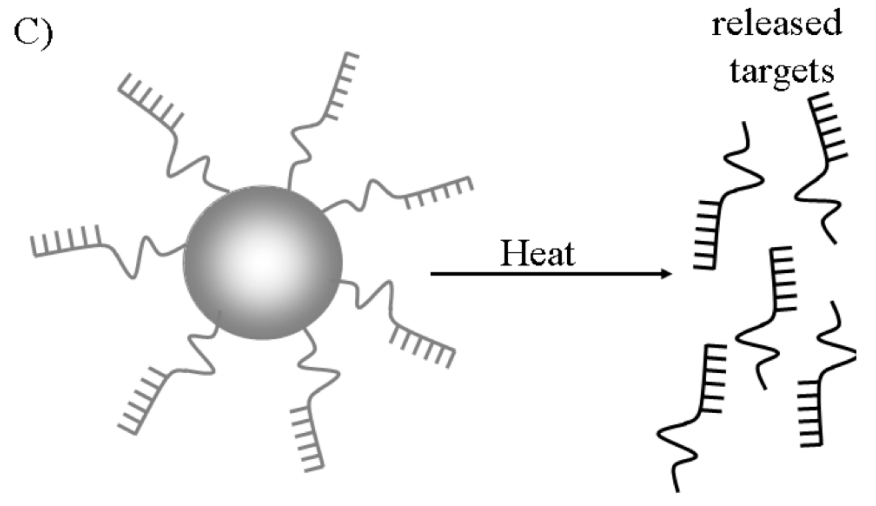

Fig. (1). Conceptual representation of the enrichment approach. (A) A complex matrix containing pathogenic and interfering nucleic acids is mixed with a solid phase prepared with specific capture probes. (B) The solid phase and the targets are subject to a stepwise temperature treatment consisting of a denaturing step at high temperature $\left(\sim 95^{\circ} \mathrm{C}\right)$ followed by an annealing step at a lower temperature in which the targets hybridize to the capture probes and the background material is eliminated. (C) The targets are released (eluted) from the solid phase through heat denaturation and subject to specific PCR for analysis.

dom capture probes synthesis. The random capture probes were prepared in a $15 \mu 1$ volume containing $40 \mathrm{mM}$ Tris- $\mathrm{HCl}$ (pH 7.5), $20 \mathrm{mM} \mathrm{MgCl}, 40 \mathrm{mM} \mathrm{NaCl}, 20 \mu \mathrm{M}$ primer $\mathrm{D}, 67$ $\mu \mathrm{M}$ dNTPs, $4 \mathrm{U}$ of Sequenase DNA polymerase version 2.0 (USB corporation, Cleveland, OH), and $7 \mathrm{ul}$ of the prepared templates with preliminary denaturation at $95^{\circ} \mathrm{C}$ for $2 \mathrm{~min}$. followed by incubation at $10^{\circ} \mathrm{C}$ for $5 \mathrm{~min}$, then $37^{\circ} \mathrm{C}$ for 20 min. Sequenase DNA polymerase and dNTPs were added after the denaturation step. All the amplified products were purified by incubating with $5 \mu 1$ of ExoSAP-IT (USB) at $37^{\circ} \mathrm{C}$ for $30 \mathrm{~min}$., then passing through ProbeQuant G-50 Micro 
Columns (Amersham Biosciences, Piscataway, NJ) to remove unused primers and nucleotides, and stored at $4^{\circ} \mathrm{C}$ until used. The amplified products were electrophoresed on $1.2 \% \mathrm{TAE}$ agarose gels and visualized by ethidium bromide staining. The concentration of the PCR products was determined with a NanoDrop ND-1000 UV/Vis spectrophotometer (NanoDrop Technologies, Wilmington, DE).

Specific capture probe synthesis. The primers were synthesized with a 5 ' amino modifier and 6 carbon spacer (Supplemental Table 1). Amplification reactions were carried out in a $50 \mu \mathrm{l}$ volume containing $20 \mathrm{mM}$ Tris- $\mathrm{HCl}$ (pH 8.4), $50 \mathrm{mM}$ $\mathrm{KCl}, 3 \mathrm{mM} \mathrm{MgCl}$, $200 \mu \mathrm{M}$ dNTPs, $2 \mu \mathrm{M}$ each forward and reverse primer, $2 \mathrm{U}$ of Platinum Taq DNA Polymerase (Invitrogen) and $1 \mathrm{ng}$ of template. The amplification reaction was carried out with preliminary denaturation at $95^{\circ} \mathrm{C}$ for $3 \mathrm{~min}$. followed by 40 cycles of: $94^{\circ} \mathrm{C}$ for $30 \mathrm{sec}$., $50^{\circ} \mathrm{C}$ for $30 \mathrm{sec}$., and $72^{\circ} \mathrm{C}$ for $60 \mathrm{sec}$. The products were purified using Qiagen PCR purification kit (Qiagen) and eluted in Tris-HCl buffer (pH 8.4).

Solid phase preparation and probe immobilization. Fabrication of solid phase and immobilization of capture probes were carried out as previously described [13]. Unless otherwise noted, the following term "specific-flu beads" refers to magnetic beads functionalized with capture probes specific for hemagglutinin (HA), neuraminidase (NA), and matrix genes of FluA H1N1; "specific-Ad4 beads" refers to beads functionalized with capture probes for the fiber, hexon and E1A genes of HAdV-4; "random-flu beads" refers to beads functionalized with random capture probes for FluA H1N1 and "random-Ad4 beads" refers to beads functionalized with random capture probes for $\mathrm{HAdV}-4$. The term $\mathrm{G}(0.5)$ beads refer to magnetic microbeads functionalized with a generation 0.5 phosphorous dendrimer respectively.

Target preparation. For the single pathogen enrichment experiments the target FluA H1N1 cDNA was prepared by single step RT-PCR as described previously. HAdV-4 DNA was used intact or fragmented by endonuclease digestion using EcoRI (New England Biolabs) which resulted in 4.3 to 16 $\mathrm{kb}$ fragments. Targets for the enrichment from complex mixtures were prepared by mixing $200 \mathrm{ng}$ of human genomic DNA (Roche Applied Sciences, Indianapolis, IN), $500 \mathrm{ng}$ of Control RNA (Ambion Inc., Austin, TX), 1 ng of FluA H1N1 RNA and 1 ng of HAdV-4 DNA. First strand cDNA synthesis was performed using 400 U of SuperScript III Reverse Transcriptase (Invitrogen Life Technologies, Carlsbad, CA) and primer LN at $8.3 \mu \mathrm{M}$ with $25 \mathrm{mM}$ of each dNTP according to the manufacturer's recommended protocol.

Enrichment of selected targets. Prior to sequence-capture, the beads were washed once with $100 \mathrm{ul}$ of $0.1 \%$ SDS/1XSSC and incubated at $55{ }^{\circ} \mathrm{C}$ for 10 minutes. The supernatant was removed and sequence-capture was performed by mixing the prepared solid phases with the targets in a buffer containing $10 \mathrm{XSSC} / 3 \% \mathrm{SDS}$ followed by three post-hybridization washes as described previously [13]. After the post hybridization wash, the beads were washed once with $100 \mu$ l of the low salt buffer $(0.1 \%$ Tween $20 / 0.1 \mathrm{X}$ SSC) and the supernatant discarded. The beads were re-suspended in $22 \mu \mathrm{l}$ of the low salt buffer, incubated at $95^{\circ} \mathrm{C}$ for variable periods of time and the supernatant recovered. When required, a second elution was performed immediately after the first one by re-suspending the beads in $22 \mu 1$ of nuclease free water pre-warmed at $95^{\circ} \mathrm{C}$ and incubating them at $95^{\circ} \mathrm{C}$ for variable periods of time. The captured targets were eluted from the solid phase through heat denaturation at $95^{\circ} \mathrm{C}$ using a low salt buffer and nuclease free water. The recovery of the captured targets was addressed qualitatively and semi-quantitatively using specific PCR (primers are listed in supplemental Table 2), gel electrophoresis and UV spectroscopy.

\section{RESULTS}

The development of a single-step sequence-capture solid phase is a complex process with numerous variables that can affect the hybridization and the elution steps. These variables can be separated in three categories: 1) solid phase properties, namely linker size, number of covalent attachment sites and surface area; 2) target characteristics, such as length, base pair composition, conformation and diffusivity; and 3) capture probe characteristics, that is, probe length, selectivity and specificity. The quantitative evaluation of each variable separately would require a tremendous experimental and bioinformatics effort which is out of the scope of the present study. As a start, we designed a simple, yet insightful set of experiments to demonstrate the feasibility of a single-step process and to delineate the issues relevant in the development of this type of solid phases. This concept is schematically presented in Fig. (1). Since the enrichment of a pathogen depends on its genome characteristics (single-strand $v s$ double strand, base composition, length and composition), the capture conditions of each pathogen are tested independently and then a common set of conditions for attempting simultaneous capture will be chosen.

Enrichment of FluA H1N1 cDNA using G(0.5) solid phase: This initial set of experiments was performed using a generation 0.5 phosphorous dendrimer $(1.5 \mathrm{~nm}$ size, 6 branches for covalent binding, $\mathrm{G}(0.5))$ with capture probes for $H A, N A$, and matrix of FluA H1N1 and pure FluA H1N1 templates to allow the optimization of the hybridization and the elution conditions. For this purpose we tested different buffer compositions $(0.1 \%$ SDS/1XSSC, $0.05 \%$ SDS $/ 1$ XSSC and $0.1 \%$ SDS/0.1XSSC, all at $\mathrm{pH}$ 7.5), temperatures and incubation times. Our results indicated that an initial $95^{\circ} \mathrm{C}$ denaturation step for 10 minutes followed by an incubation step at $70^{\circ} \mathrm{C}$ for 5 minutes prior to annealing of the targets at $50^{\circ} \mathrm{C}$ for 35 minutes with a slow transition in between steps (ramp rate of $0.1{ }^{\circ} \mathrm{C} / \mathrm{s}$ ) showed the best capture efficiency (Fig. 2). Given the thermal stability of the solid phase, heat denaturation at $95^{\circ} \mathrm{C}$ was used to elute the targets. Experiments performed on elution series under different conditions (time and elution buffers) showed that most of the target was released during the first and the second elution steps, thus two elution steps with incubation time of 20 minutes at different stringency conditions were used. The first elution was performed using the low salt elution buffer and the second one in nuclease-free water. The long elution time should provide the target with more time to diffuse away from the solid phase into the solution, and the lack of monovalent ions in the second elution step (water) would facilitate the denatured targets to remain unbound and away from the captured probes. Fig. (2) shows a representative image of the PCR products on the recovered targets from three independent sequence capture experiments. 


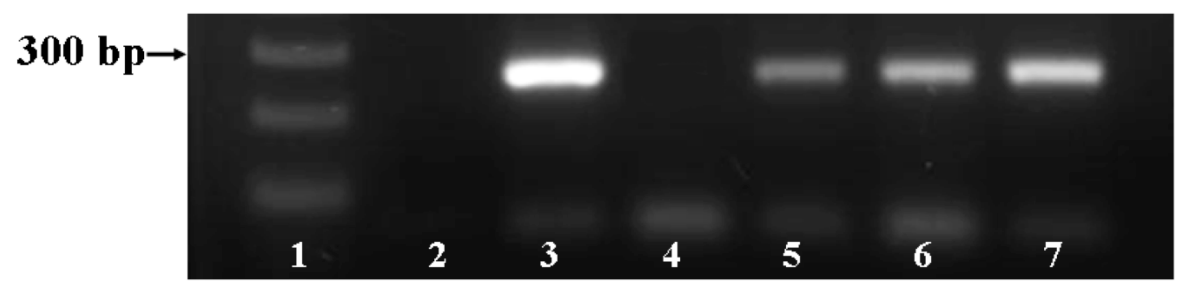

Fig. (2). Representative image of PCR products on recovered influenza A H1N1 cDNA targets after enrichment using G(0.5) specific-flu beads. Lane 1, molecular weight marker; lane 2, negative control; lane 3, positive control; lane 4, eluted product of a blank target; lanes 5-7, eluted products of three identical enrichment experiments performed with specific-flu beads.

For this set of experiments, only half of the elution volume was used in the PCR reaction. Quantification of the purified PCR products using UV spectroscopy showed a recovery between 40 and $65 \%$ with respect to the PCR positive control (1ng of FluA H1N1 cDNA). The shedding of capture probes from the beads can create artifacts as documented by Koblizkova et al [5]. As a control, a blank sample was subject to the same process in which no artifacts were observed (Fig. 2, lane 4.) which indicates that the dendrimer-capture probe linkage was strong enough to withstand the elution conditions as previously demonstrated [13]. Another control to indicate non-specific adsorption as well as no nonspecific probe-target hybridization with other pathogen was performed using samples spiked with HAdV-4. The HAdV-4 was recovered only from the hybridization supernatant and none was present in the elutions (data not shown). The difference in the yields between the three samples is due to experimental variation. Similar experiments using random capture probes showed an average recovery of $50 \%$, with less variation.

Enrichment of HAdV-4 using a $G(0.5)$ solid phase: For the enrichment of HAdV-4, we performed a similar set of experiments as the ones described for the FluA H1N1 using specificAd4 beads and random-Ad4 beads. We first tested whether the enrichment conditions used for FluA H1N1 would be suitable for enrichment of HAdV-4. We found two differences in the conditions, first the intermediate $70^{\circ} \mathrm{C}$ incubation step did not have a significant effect on the capture efficiency of the HAdV-4 and second, the release of the target occurred at different proportions during the first and the second elution steps. Based on the assumption that heat denaturation at higher $\mathrm{pH}$ and low salt conditions would facilitate the release of the captured targets, we found that use of a Tris- $\mathrm{HCl}$ based buffer at pH 8 improved the recovery of HAdV-4 by $\sim 10 \%$ but these conditions were not suitable for the FluA enrichment. Thus, we pursued the remaining of the experiments using the low salt elution buffer and the nuclease free water. The enrichment efficiency of HAdV-4 was tested using the optimized conditions with specific and random-Ad4 beads and the effect of target size on capture efficiency was examined by using both intact and fragmented (restriction enzyme digested) targets. The recovery observed using the random-Ad4 beads varied significantly; we observed yields ranging from 10 up to $80 \%$ regardless of whether the target was intact or fragmented (data not shown). The large variation obtained with the random-Ad4 beads results was probably due to the variations in the yield and the immobilization efficiency of the random capture probes. The most consistent results were obtained with the specific-Ad4 beads using intact targets, for this reason we pursued the remaining experiments using specific capture probes. Table 1 presents the results obtained for HAdV-4 enrichment.
For these set of experiments, only half of the elution volume was used in the PCR reactions.

Table 1. Percentage of HAdV-4 Recovered Using Specific and Random-Ad4 Beads

\begin{tabular}{|c|c|}
\hline & Recovery \\
\hline \hline Random-Ad4 beads & $10-80 \%$ \\
\hline Specific-Ad4 beads & $20-35 \%$ \\
\hline
\end{tabular}

Calculated with Respect to a PCR Positive Control (1ng HAdV-4). Only Half of the Elution Volume was Used in the PCR Reaction and the Quantification was Performed on Purified Products Through UV/VIS Spectrophotometry.

Simultaneous enrichment of FluA H1N1 and HAdV-4: Simultaneous enrichment of more than one pathogen means mixture of solid phases with various capture probes (multipathogen solid phase). To test whether the performance could be affected by the presence of non specific capture probes we examined two different solid phases, one by mixing equal amounts of specific-Ad4 beads and specific-flu beads and a second one using specific-Ad4 beads and random-flu beads. The experiments were carried out as described in the previous section and the results are presented in Table 2. As before, only half of the elution volume was used for the PCR reactions.

Table 2. Percentage of FluA H1N1 Recovered Using a MultiPathogen Solid Phase

\begin{tabular}{|c|c|}
\hline & Recovery \\
\hline \hline Specific-Ad4 beads + random-flu beads & $50 \%$ \\
\hline Specific-Ad4 beads + specific-flu beads & $19 \%$ \\
\hline
\end{tabular}

The Values are Calculated with Respect to a PCR Positive Control (1ng of FluA H1N1 cDNA). Only Half of the Elution Volume was Used in the PCR Reaction and the Quantification was Performed on Purified Products Through UV/VIS Spectrophotometry.

As shown in Table 2, the recovery of the multi-pathogen solid phase containing the random-flu beads was $50 \%$ with respect to the positive control (comparable to the recovery of the random-flu beads alone) while the recovery of the one with the specific-flu beads was only $19 \%$. The presence of non-specific solid phase (specific-Ad4 beads) did affect the capture efficiency of specific Flu beads. However, the low recovery observed when specific-flu beads are in the mixture may be due to the greater variation in performance of these beads. Since the length of the capture probes used for these experiments is similar, the variations probably resulted from differences in the probes annealing conditions. 
To evaluate the simultaneous capture of two targets, two identical experiments using the multi-pathogen solid phase prepared with specific-Ad4 beads and random-flu beads were performed. Despite the different requirement for enriching FluA H1N1 and HAdV-4, we decided to test the simultaneous enrichment of both pathogens using the multi-pathogen solid phase under the conditions optimized for the FluA H1N1 given that the incorporation of the $70^{\circ} \mathrm{C}$ incubation step does not have any detrimental effect on the HAdV-4. For the analysis, each elutant was split in half to perform PCR for each pathogen. Since this would correspond to $1 / 4$ of the volume used for the single pathogen experiments we increased the amount of target used to $2 \mathrm{ng}$. As before, a blank target was included in each experiment to confirm the lack of probe shedding. Table 3 presents the overall recovery of each pathogen and the fraction from the total that was recovered in each elution step. The first elution (E1) was performed using the low salt buffer and the second elution (E2) using nuclease free water. As before, both elution steps were carried out at $95^{\circ} \mathrm{C}$ for 20 minutes. The enrichment yield for both pathogens is comparable with the single pathogen experiment.

Table 3. Percentage Recovered of FluA H1N1 and HAdV-4 after Enrichment Using a Multi-Pathogen Selective Solid Phase from Two Independent Experiments

\begin{tabular}{|c|c|c|}
\hline & Experiment 1 & Experiment 2 \\
\hline \hline Flu A Total & $40 \%$ & $45 \%$ \\
\hline Fraction of Flu A recovered in E1 & $20 \%$ & $50 \%$ \\
\hline Fraction of Flu A recovered in E2 & $80 \%$ & $50 \%$ \\
\hline Ad4 Total & $30 \%$ & $35 \%$ \\
\hline Fraction of Ad4 recovered in E1 & $90 \%$ & $10 \%$ \\
\hline Fraction of Ad4 recovered in E2 & $10 \%$ & $90 \%$ \\
\hline
\end{tabular}

The Total Amounts are Calculated with Respect to the PCR Positive Control (1 ng) and the Terms E1 and E2 Denote First and Second Elution Respectively. The Percentages of E1 and E2 Indicate the Fraction of the Total Amount Recovered in Each Step. The Quantification was Performed by UV Spectroscopy After Purification of the PCR Products.

To further test the enrichment efficiency of both pathogens in the presence of complex matrices, this protocol was used to test the simultaneous enrichment of HAdV-4 and FluA H1N1 (Fig. 3). For this purpose, a mixture of background nucleic acids composed of ribosomal RNA (rRNA) and human genomic DNA (hgDNA) were spiked with HAdV-4 and FluA H1N1 RNA. This pool of nucleic acids was then subject to a single step reverse transcription using a random primer (primer LN). The products were then enriched using a multipathogen solid phase prepared with random flu and specific Ad4-beads. The capture efficiency was significantly lower in comparison with the experiments performed without background, no more than $10-15 \%$ recovery (data not shown). The low enrichment efficiency is probably due to the non-specific interaction between the probes and background material, and the current protocol has not worked out yet the optimal conditions to reduce or eliminate these interactions.

\section{DISCUSSION}

We have demonstrated the use of a magnetic bead based solid phase for sequence-capture (enrichment) of pathogenic nucleic acids in a single step. The thermal stability required in the solid phase to perform enrichment in a single step and the recovery of the captured targets through heat denaturation was achieved using a phosphorous dendrimer as a linker. Our results suggest that the capture efficiency of influenza A H1N1 (FluA H1N1) and adenovirus serotype 4 (HAdV-4) depended on the probe and target characteristics (single-strand $v s$ double strand DNA, length and base composition), the hybridization conditions (buffer composition, temperature and time), as well as the elution conditions. We also investigated the feasibility of a multi-pathogen solid phase for enrichment of targets from complex matrices containing human genomic DNA and rRNA in an 800 fold excess with respect to the targets. The results presented in this work demonstrate for the first time the feasibility to perform enrichment of multiple low abundance targets in a single step using a selective solid phase.

We demonstrated that it is possible to use a mixed solid phase to enrich both targets simultaneously. In comparison with the single pathogen solid phases, a multi-pathogen enrichment process is more complicated and our results demonstrate that this process is plausible but requires a careful tuning of the hybridization step and the elution step. Although the enrichment yields is much lower in the presence of background materials, it should be noted that the enrichment is occurring in the presence of 800 fold excess of background material and this enrichment process actually raise the target/background ratio which may be sufficient for some of the downstream detection methods. The decrease in the enrichment yield in the presence of background material suggests that the background material is interfering with the capture of the targets by hybridizing non-specifically to the capture probes. Other factors, such as linker type, temperature and diffusion time can also affect the specificity of the solid phase. Indeed, our current ongoing work indicates that the hybridization temperature and diffusion time may play a critical role in determining the non-specific interactions. For instance, experiments performed with a generation 4.5 dendrimer (G 4.5) showed an enhanced performance in the capture of the targets with less non-specific interactions. Although these results are preliminary, we believe that these results pave the way to further improvement of the enrichment process.

Given the difference of genome characteristics of the two pathogens tested, it is not surprising to find that the capture conditions for FluA H1N1 and HAdV-4 vary. For FluA $\mathrm{H} 1 \mathrm{~N} 1$, an intermediate incubation step is critical to the capture efficiency; its omission led to a lower enrichment yield. However, this incubation step has no effect for HAdV-4 enrichment. The plausible explanation for this phenomenon is the diffusion constraints of the targets during the hybridization and the elution might be dominating factor. The other factor needs to be considered is the nature of the single-strand vs double-stranded DNA. The incorporation of a $70^{\circ} \mathrm{C}$ incubation step probably helps denaturing the secondary structure of FluA H1N1 cDNA and enhances the probes-targets interaction, while HAdV-4 (double-strand DNA) can re-anneal very quickly with its complementary strand [15] and renders this step ineffective. In addition, even though excess probes were present in the experiments presented here, it is possible that more probes (200 fold excess) are needed for HAdV-4 (double-strand DNA) to compete with the re-annealing of the complementary strands and increase the enrichment efficiency. The higher yield of FluA H1N1 suggested that the smaller target size provided better capture efficiency. The en- 


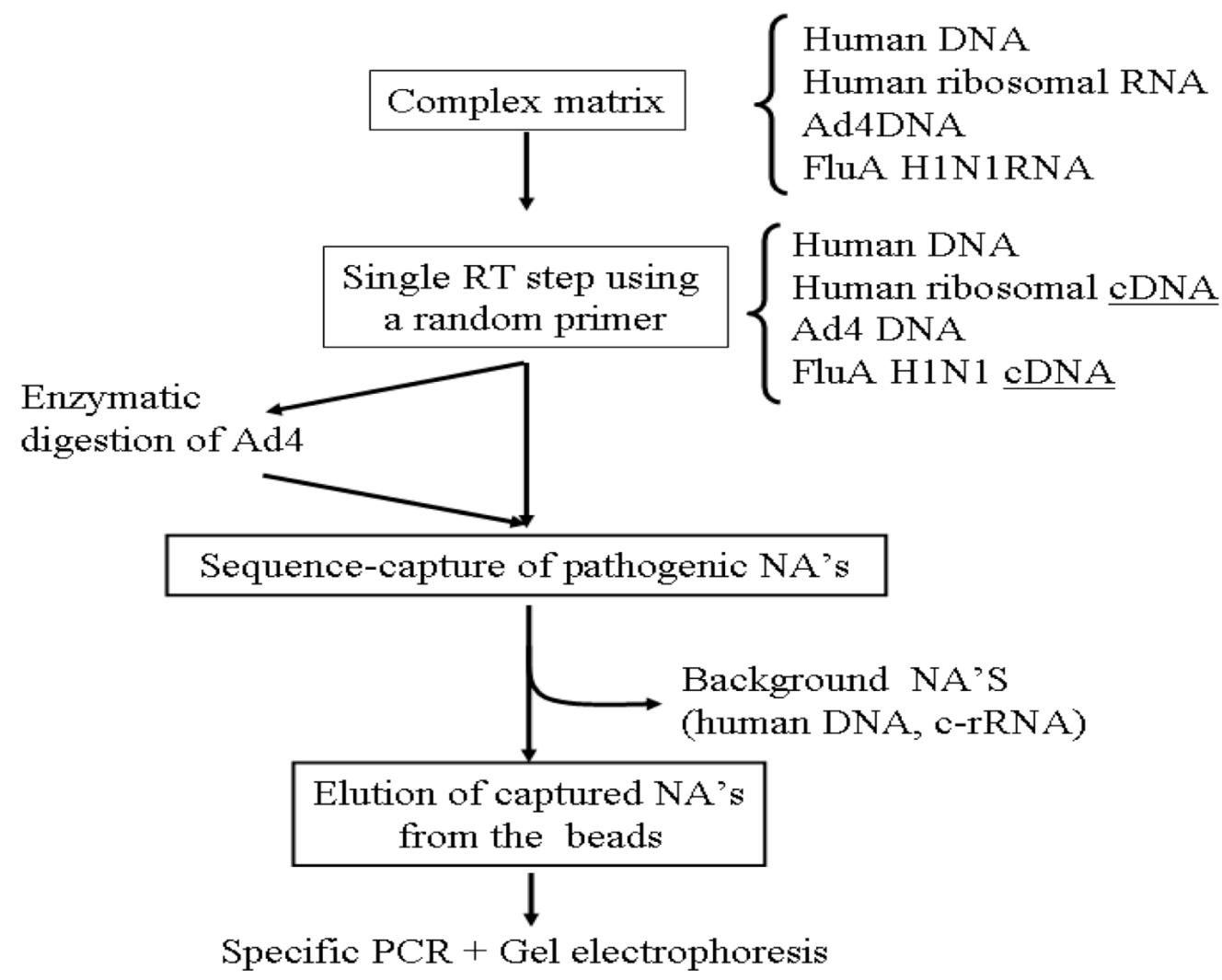

Fig. (3). Schematic representation of the protocol used to test the simultaneous enrichment of FluA H1N1 RNA and HAdV-4 from a complex mixture. Reverse transcription (RT) was performed using primer LN. The RT product was then mixed with the solid phase and the hybridization buffer (10X SSC/10\% SDS) to perform sequence capture. Elution of the captured nucleic acids (NA's) was performed using two consecutive heat denaturing steps at different ionic strength conditions. The recovered targets were analyzed through specific amplification and gel electrophoresis.

zymatic cleavage (to reduce the genome size) of HAdV-4 did not increase the enrichment efficiency neither in the single pathogen experiments nor the mixed solid phase. This is probably due to the fact that the fragment sizes generated (4.3$16 \mathrm{~kb}$ ) are still much longer than targeted FluA H1N1 segmented cDNAs (ranged from 0.8 to $1.7 \mathrm{~kb}$ ). Further investigation with combination of restriction enzymes for HAdV-4 digestion will be needed to investigate whether smaller targets could increase the enrichment efficiency and how the specific cleavage site affects the capture efficiency.

Another important factor that affects the enrichment process is the capture probes. The difference of enrichment efficiency observed between the specific and random capture probes for FluA H1N1 and HAdV-4 is probably a result of differences in the probe sequence and length. However, it is not clear why the opposite effect observed for capturing HAdV-4 and FluA H1N1. Up to this point, we have very limited understanding of the capture probes used since the random probes are not restricted to a particular gene but rather target several parts of the genome, and the length of these probes comprises a range between $\sim 100$ and 600 bases long which in turn makes characterization of the probes behavior almost impossible. As for the specific probes, due to limited number of specific probes used, it is difficult to draw any conclusion. Future work will focus on better understanding of the probes used with careful design of the capture probes using bioinformatics analysis to ensure the selectivity. The inconsistency in the amount of HAdV-4 recovered during the first and the second elution steps between experiments is possibly related to the size of the targets and how easily they denature and diffuse away from the solid phase during each elution step. Under the conditions used for these experiments the released targets are single stranded, in a low salt or salt free media (elution buffers) and very likely to acquire a secondary structure which affects their diffusion into the elutant. Indeed, during the course of these experiments we found that a key factor in increasing the enrichment yield was to briefly resuspend the beads every 10 minutes during the elution steps which might be facilitating the movement of the released targets from the solid phase into the solution.

\section{CONCLUSION}

While the yield is not yet optimal, we have demonstrated the feasibility to enrich multiple targets in a single step using selective solid phases. This study identified and evaluated some critical issues associated with the development of a selective solid phase for single step enrichment. A "multipathogen" solid phase was also demonstrated to be feasible for the simultaneous enrichment of FluA H1N1 and HAdV-4 from a matrix containing an 800 excess fold of background material. Our results suggest that the characteristics of the probes and the assay conditions (temperature, time and buffer composition), as well as the targets (length and conformation) play significant roles in the optimization of the enrichment process. Understanding these variables is critical for the optimization of the enrichment efficiency, especially in the presence of background material, an interference of the process. 


\section{ACKNOWLEDGEMENTS}

This work was funded by the Office of Naval Research. We thanks Drs. Jinny Liu and George Anderson for critically reviewing the paper. The opinions and assertions contained herein are those of the authors and are not to be construed as those of the U. S. Navy or military service at large.

\section{ABBREVIATIONS}

$$
\begin{array}{ll}
\text { FluA H1N1 } & \text { Influenza A/H1N1 } \\
\text { HAdV-4 } & =\text { Human adenovirus serotype } 4
\end{array}
$$

\section{REFERENCES}

[1] Boni, J.; Shah, C.; Flepp, M.; Luthy, R.; Schupbach, J. J. Med. Virol., 2004, 72, 1-9.

[2] Brugiere, O.; Vokurka, M.; Lecossier, D.; Mangiapan, G.; Amrane, A.; Milleron, B.; Mayaud, C.; Cadranel, J.; Hance, A. J. Am. J. Respir. Crit. Care Med., 1997, 155, 1478-81.

[3] Mangiapan, G.; Vokurka, M.; Schouls, L.; Cadranel, J.; Lecossier, D.; van Embden, J.; Hance, A. J. J. Clin. Microbiol., 1996, 34, 1209-15.
[4] Millar, D. S.; Withey, S. J.; Tizard, M. L.; Ford, J. G.; HermonTaylor, J. Anal. Biochem., 1995, 226, 325-30.

[5] Koblizkova, A.; Dolezel, J.; Macas, J. Biotechniques, 1998, 25, 324.

[6] Marsh, I.; Whittington, R.; Millar, D. Mol. Cell Probes, 2000, 14, 219-32.

[7] Roring, S.; Hughes, M. S.; Skuce, R. A.; Neill, S. D. Vet. Microbiol., 2000, 74, 227-36.

[8] Vansnick, E.; de Rijk, P.; Vercammen, F.; Rigouts, L.; Portaels, F.; Geysen, D. Vet. Microbiol., 2007, 122, 166-71.

[9] Muir, P.; Nicholson, F.; Jhetam, M.; Neogi, S.; Banatvala, J. E. J. Clin. Microbiol., 1993, 31, 31-8.

[10] Roring, S.; Hughes, M. S.; Beck, L. A.; Skuce, R. A.; Neill, S. D. Vet. Microbiol., 1998, 61, 71-80.

[11] Fan, Z. H.; Mangru, S.; Granzow, R.; Heaney, P.; Ho, W.; Dong, Q.; Kumar, R. Anal. Chem., 1999, 71, 4851-4859.

[12] Waner, M. J.; Navrotskaya, I.; Bain, A.; Oldham, E. D.; Mascotti, D. P. Biophys. J., 2004, 87, 2701-13.

[13] Archer, M. J.; Lin, B.; Wang, Z.; Stenger, D. A. Anal. Biochem., 2006, 355, 285-97.

[14] Stone, B.; Burrows, J.; Schepetiuk, S.; Higgins, G.; Hampson, A.; Shaw, R.; Kok, T. J Virol. Methods, 2004, 117, 103-12.

[15] Church, R. B.; Georgiev, G. P. Mol. Biol. Rep., 1973, 1, 21-5.

(C) Archer et al.; Licensee Bentham Open.

This is an open access article distributed under the terms of the Creative Commons Attribution License (http://creativecommons.org/license/by/2.5/), which permits unrestrictive use, distribution, and reproduction in any medium, provided the original work is properly cited. 
Supplemental Table 1. Primer Sequences for Capture Probe Synthesis

\begin{tabular}{|c|c|}
\hline Primer D & 5'- $\mathrm{NH}_{2}$ GTT TCC CAG TAG GTC TCN NNN NNN N-3' \\
\hline Primer L & 5'-CGA TAC GAC GGG CGT ACT AGC G-3' \\
\hline Primer LN & 5'-CGA TAC GAC GGG CGT ACT ACG GNN NNN NNN N-3' \\
\hline FluA N1 For & 5'- $\mathrm{NH}_{2}$ GGG TTA GCC ACT CAA TCC AA-3' \\
\hline FluA N1 Rev & $5^{\prime}-\mathrm{NH}_{2}$ TAT AGC CCA CCC ACG GAT AG-3' \\
\hline FluA H1 For & 5'- $\mathrm{NH}_{2}$ GCA CCA ATG GAT GAA TGT GA-3' \\
\hline FluA H1 Rev & 5'-NH ${ }_{2}$ CAA TGG CTC CAA ACA AAC CT-3' \\
\hline FluA MAT For & 5'- $\mathrm{NH}_{2}$ GAC CAA TCC TGT CAC CTC TGA-3' \\
\hline FluA MAT Rev & 5'- $\mathrm{NH}_{2}$ GTA TAT GAG KCC CAT RCA ACT-3' \\
\hline Ad4 Hex For & 5'-NH ${ }_{2}$ CGC TTC GGA GTA CCT CAG TC-3' \\
\hline Ad4 Hex Rev & 5'-NH ${ }_{2}$ AGC GTG TAG CGC ACT TTG TA-3' \\
\hline Ad4 Fib For & 5'-NH ${ }_{2}$ GAC TCG GGA AAA CTC ATT GC-3' \\
\hline Ad4 Fib Rev & 5'- $\mathrm{NH}_{2}$ AAA CCT GAG CCA AAA GCT GA-3' \\
\hline Ad4 E1A For & 5'-NH ${ }_{2}$ GAT TTG CTT CAG GGT GGA GA-3' \\
\hline Ad4 E1A Rev & 5'-NH ${ }_{2}$ CAC AGG TCT GCA CCT GCT TA-3' \\
\hline
\end{tabular}

Supplemental Table 2. Primer Sequences for Analysis of Enriched Targets

\begin{tabular}{|c|c|}
\hline Flu Amp Forward & 5'-GAC CAA TCC TGT CAC CTC TGA-3' \\
\hline Flu Amp Reverse & 5'-GTA TAT GAG KCC CAT RCA ACT-3' \\
\hline Ad4-F6 Forward & 5'-GGAATAGATTCAACTTCTGGAACTG-3' \\
\hline Ad4-R5 Reverse & 5'- TCTGGATCGTAGTTAGCAACAATAG-3' \\
\hline
\end{tabular}

